Revista del Centro de Investigación de la Universidad La Salle

Vol. 14, No. 55, Enero-Junio, 2021: 179-195

DOI: http://doi.org/10.26457/recein.v14i55.2830

\title{
Asociación entre índices nutricionales y días de ventilación mecánica en adultos mayores críticamente enfermos
}

\section{Association between nutritional indeces and days of mechanical ventilation in critically ill older adults}

\author{
Luis Alberto Martínez Palma* \\ Centro Médico Dalinde y Facultad Mexicana de Medicina, Universidad La Salle \\ México (México) \\ Diana Enríquez Santos \\ Centro Médico Dalinde y Facultad Mexicana de Medicina, Universidad La Salle \\ México. (México) \\ Paolo Alberti Minutti \\ Centro Médico Nacional Siglo XXI, IMSS y Facultad Mexicana de Medicina, \\ Universidad La Salle México (México)
}

Recibido: 12 de noviembre de 2020

Aceptado: 14 de abril de 2021

Publicado: 31 de mayo de 2021

\section{Resumen}

Objetivo: Evaluar la relación entre las escalas GNRI y CONUT al ingreso y la presencia de ventilación mecánica prolongada en adultos mayores críticamente enfermos.

Diseño y metodología: Se realizó un estudio transversal, retrospectivo, en la UCI del Centro Médico Dalinde, entre marzo 2019 y febrero 2020, fueron seleccionaron sujetos $>60$ años que requirieron ventilación mecánica al ingreso, se formaron dos grupos: Grupo $\mathrm{A}<7$ días de ventilación mecánica y Grupo B > 7 días de ventilación, se registraron variables demográficas, diagnósticos de ingreso, días de estancia en la UCI, comorbilidades preexistentes y para valorar el estado nutricional se utilizaron GNRI y CONUT. Se empleó estadística descriptiva $\chi 2$ y prueba de Fisher, las variables continuas con prueba de $\mathrm{t}$ de

"Email: palma23.ap@gmail.com

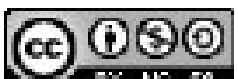

Revista del Centro de Investigación. Universidad La Salle por Dirección de Investigación. Universidad La Salle Ciudad de México se distribuye bajo una Licencia Creative Commons Atribución-NoComercial-CompartirIgual 
Student, U de Mann-Whitney según fue el caso, se consideró la significancia un valor de $\mathrm{p}<0.05$.

Resultados: 68 sujetos cumplieron con criterios de inclusión, la edad promedio fue de 81 años RIQ [7286], 51.5\% del sexo masculino, la mediana de días de estancia en la UCI en el grupo A fue de 8 días y 20 días para el grupo B, la puntuación de GNRI para el grupo A fue 90 [74-97], el grupo B 84 [75-93] $(p=0.432)$, la escala de CONUT para el grupo A 8 [6-9] y el grupo B 9 [7-11] $(p=0.135)$.

Limitaciones: tipo de estudio y número de muestra limitan la interpretación de los resultados.

Originalidad: contribuye al conocimiento de la evolución de los adultos mayores ingresados a la UCI que reciben ventilación mecánica y su relación con la desnutrición.

Conclusiones: La relación entre estado nutricional y días de ventilación mecánica; está modulada por múltiples variables. El uso de GNRI y CONUT por sí solos no es suficiente para predecir si el adulto mayor se someterá a ventilación mecánica prolongada.

Palabras clave: Cuidados Intensivos; Geriátrico; CONUT; GNRI 


\section{Abstract}

Objective: evaluate the relationship between the GNRI and CONUT scales at admission and the presence of prolonged mechanical ventilation in critically ill older adults.

Design and methodology: A cross-sectional, retrospective study was carried out in the ICU of Centro Medico Dalinde, between March 2019 and February 2020, subjects $>60$ years old who required mechanical ventilation on admission were selected, two groups were formed: Group A $<7$ days of mechanical ventilation and Group B $>7$ days of ventilation, demographic variables, admission diagnoses, days of stay at the ICU, preexisting comorbidities and to assess nutritional status were used GNRI and CONUT scales. Descriptive statistics $\chi 2$ and Fisher's test were used, continuous variables with Student's t test, Mann-Whitney $U$, as the case may be, significance was considered at a value of $\mathrm{p}<0.05$.

Results: 68 subjects met the inclusion criteria, the average age was 81 years old IQR [72-86], 51.5\% male, the median days of stay in the ICU in group A was 8 days and 20 days for group B, the GNRI score for group A was 90 [74-97], group B 84 [75-93] ( $\mathrm{p}=0.432)$, the CONUT scale for group A 8 [6-9] and group B 9 [7-11] $(\mathrm{p}=0.135)$.

Limitations: the type of study and sample number limit the interpretation of the results.

Originality: contribute to the knowledge of the evolution of older adults admitted to the ICU who receive mechanical ventilation and its relationship with malnutrition.

Conclusions: The relationship between nutritional status and days of mechanical ventilation; it is modulated by multiple variables. The use of GNRI and CONUT alone is not sufficient to predict whether the elderly will undergo prolonged mechanical ventilation.

Keywords: Intensive Care; Geriatric; CONUT; GNRI 


\section{Introducción}

La población está experimentando un proceso de envejecimiento, México ha incrementado su esperanza de vida de 44 años en la década de los cuarenta, a 75,2 años en el 2016; se espera que para el 2050 uno de cada cuatro mexicanos sea mayor de 60 años lo que represente cerca del $28 \%$ de la población. (Alfonso López-Soto et al., 2009; Carrillo-Esper R et al., 2019; Villagordoa M J., 2007)

Los adultos mayores definidos como aquellos de más de 60 años, (NOM-167-SSA1-1997; Instituto Nacional de las Mujeres., 2015) son considerados una población en alto riesgo de desarrollar problemas de salud y que requieren necesidades específicas. Poseen una alta prevalencia de enfermedades crónico- degenerativas y debido a su evolución; es probable que en algún momento presenten desordenes o agudizaciones que requieran manejo médico o quirúrgico y que ameriten su ingreso a una Unidad de Cuidados Intensivos (UCI). (Carrillo-Esper R et al., 2019; Sánchez Zúñiga M J et al., 2018; Villagordoa M J., 2007)

En los países desarrollados los pacientes mayores de 65 años representan entre el 25-51\% del total de ingresos a la UCI, donde consumen hasta el 60\% de las estancias hospitalarias, (Le Maguet P et al., 2014) aunque en nuestro país no contamos con estadísticas al respecto; los ancianos tienen un riesgo aumentado de caer en situación de dependencia después de su ingreso a la UCI debido a la fragilidad que presentan. (Angus D. C., 2017; Reyes J. C et al., 2016)

Un rasgo importante en este grupo etario es la detección de desnutrición y la pérdida de peso involuntaria, mismas que se han asociado a un incremento en la mortalidad a los dos años del egreso hospitalario (RR 2.43; IC 95\% de 1.34 a 4.41), (Wallace J. I. et al., 1995) hay evidencia para considerar a la nutrición como una estrategia terapéutica capaz de alterar positivamente el desenlace de estos pacientes sometidos a ventilación mecánica (VM), los datos disponibles en 8 estudios incluyeron 1846 pacientes, el análisis mostró que la administración temprana de alimentación hipocalórica condiciona una duración significativamente menor de dependencia al ventilador comparado con el grupo de dieta hipercalórica, mostrando una diferencia media ponderada $-0.80 ; 95 \% \mathrm{CI},-1.39$ para $-0.21, \mathrm{p}=0.0001 ; \mathrm{I} 2=11 \%, \mathrm{p}=$ 0.35) esto indica una reducción de 19.2 horas de la dependencia al ventilador. (Chelkeba L. et al., 2017; Ibrahim E. H. et al., 2002)

Se han desarrollado escalas como la GNRI (Geriatric Nutritional Risk Index) en la que se evidenció la relación de puntuación de severidad y albúmina correlacionándose positivamente $(\mathrm{p}<0.001)$, puntuaciones menores de 82 están relacionadas a un mayor riesgo de mortalidad con un Odds radio de 29.0 (IC 95\% 5.2-161.4 p < 0.001) lo que demostró que el uso de GNRI es un indicador de morbimortalidad en los ancianos. (Bouillanne O. et al., 2005) La CONUT (Controlling Nutritional Status) ha sido comparada con la Evaluación Nutricional Completa (Full Nutritional Assessment) considerada como el estándar de oro obteniendo una sensibilidad del 92.3\% y una especificidad del 85\%, (Ignacio de Ulíbarri J.et al., 2005; Suárez Núñez L. et al., 2018) ambas escalas tienen la capacidad de predecir el riesgo nutricional y morbimortalidad.

La desnutrición en los pacientes críticamente enfermos puede ocasionar disfunción diafragmática inducida por el ventilador, (Vassilakopoulos T. et al., 2004) calculándose una pérdida del 6\% del espesor 
del diafragma por cada día de ventilación, a partir de las primeras 24 a 48 horas, (Grosu H. B. et al., 2012; Hermans G. et al., 2010) hecho que repercute directamente en la capacidad pulmonar, (Grippa R. B. et al., 2017) esta disfunción muscular respiratoria secundaria a procesos proteolíticos disminuyen la masa de fibras musculares, (B. Polla. et al., 2004) lo que aumenta la necesidad de mayor tiempo bajo VM (Jung B. et al., 2011) considerándose un tiempo mayor de 7 días como ventilación mecánica prolongada por la European Respiratory Society (ERS) Task Force (Funk G. C. et al., 2010) y el Weaning according to New Definition study (WIND) (Béduneau G. et al., 2017) para evitar este efecto deletéreo de la VM se han descrito alternativas como la movilización temprana de los pacientes, disminuir las modalidades controladas del ventilador, evitar los altos niveles de sedación. El apoyo nutricional es otro elemento de la enfermedad crítica que participa en la evolución, es preciso acortar dicho periodo con la intención de mejorar la calidad de vida y disminuir los costos y los días de estancia hospitalaria. (Magda C. Díaz. et al., 2014)

Justificación: Resulta necesario analizar la asociación que existe entre el estado nutricional de los adultos mayores y la duración de la VM, la malnutrición y la duración de la VM contribuyen al pronóstico del paciente, la correcta identificación de pacientes en riesgo nutricional mejora la atención del paciente.

Objetivo primario: evaluar la asociación entre las puntuaciones GNRI y CONUT al ingreso y la presencia de ventilación mecánica prolongada en adultos mayores críticamente enfermos.

Objetivos secundarios:

1. Describir la demografía entre los adultos mayores que presentaron ventilación prolongada respecto a los que no.

2. Conocer la puntuación de GNRI y CONUT de los adultos mayores ingresados a la UCI.

\section{Material y métodos}

Se realizó un estudio transversal, retrospectivo en la UCI “Gastón Segovia Mejía” del Centro Médico Dalinde, en la Ciudad de México, entre marzo de 2019 y febrero de 2020, se seleccionaron a todos los sujetos mayores de 60 años que requirieron VM al ingreso por al menos 72 horas, se excluyeron del estudio a pacientes con diagnóstico de neumopatía preexistente.

Los datos fueron tomados de expedientes electrónicos y se registraron variables demográficas: edad y sexo, antropométricas como talla, peso e índice de masa corporal definido como la relación entre el peso y la talla al cuadrado, producto de esta operación se clasificó en peso bajo IMC $<18.5$, normal 18.524.9, 25-29.9 sobrepeso, obesidad grado I 30-34.9, obesidad grado II 35-39.9 y obesidad grado III IMC $>40$, (Nuttall F. Q., 2015) diagnóstico de ingreso a la unidad: sepsis definida como una disfunción orgánica potencialmente mortal debido a una respuesta disrregulada del huésped a la infección con un SOFA $>2$ puntos, (Mervyn Singer. et al., 2016) estado postoperatorio definido como el periodo de tiempo posterior a una intervención quirúrgica tanto en la Unidad de Cuidados Postanestésicos como las 48 horas subsecuentes, (A. J. Bowyer. Et al., 2015) patología gastrointestinal definida como sangrado de tubo digestivo alto o bajo, falla ventilatoria referida como la incapacidad del sistema respiratorio para funcionar eficazmente con un pO2 <60 mm Hg, y/o pCO2 >50 mm Hg, (Chakrabarti B. et al., 2016) Traumatismo 
craneoencefálico severo interpretado como una alteración del funcionamiento del cerebro secundario a una lesión traumática con una escala de coma de Glasgow $<8$ puntos, (Nancy Carney. Et al., 2016) Politraumatismo definido como lesiones múltiples que exceden la puntuación de gravedad $>17$ puntos que pueden inducir falla de diversos órganos y sistemas, (Rau C. S. et al., 2017) Enfermedad vascular cerebral descrita como la aparición de un déficit neurológico en algún territorio vascular del encéfalo por más de 24 horas (Abbott A. L. et al., 2017), Comorbilidades asociadas: definimos hipertensión arterial sistémica (HAS) como cifras sistólicas $>130 \mathrm{~mm} \mathrm{Hg}$ y/o diastólicas $>90 \mathrm{~mm} \mathrm{Hg}$ (Rosas-Peralta M. et al., 2016) o antecedente de HAS, Diabetes Mellitus tipo 2 (DM2) la definición se basó en un diagnóstico previo de DM2, tratamiento con antidiabéticos, o el cumplimiento de la definición propuesta por American Diabetes Association's, (American Diabetes Association., 2019) Enfermedad renal crónica referida como una tasa de filtración glomerular estimada (GFRe) menor de $60 \mathrm{ml} / \mathrm{min}$ por 1,73 metros cuadrados, que persiste durante 3 meses o más, (Thomas R. et al., 2008) Cáncer antecedente en el expediente clínico de enfermedad in situ o metastásica en los últimos 12 meses al ingreso, enfermedad tiroidea definida como hipotiroidismo diagnosticado o el consumo de levotiroxina, (Chaker L. et al., 2017) Enfermedad cognitiva definida como un deterioro cognitivo progresivo a la función mental anterior (Cunningham E. L. et al., 2015) para determinar la gravedad y mortalidad en la UCI, se utilizaron las escalas de riesgo APACHE II (Acute Physiology and Chronic Health disease Classification System II), SOFA (Sequential Organ Failure Assessment Score)

\subsection{Evaluación del estado nutricional}

Se estimó el estado nutricional mediante dos escalas: CONUT que clasifica la desnutrición tomando en cuenta valores de albúmina sérica, conteo total de linfocitos y los valores de colesterol total [Tabla 1]. (J.L. de-León-Rendón. Et al., 2020; López Espuela F. et al., 2019; Suzuki S. et al., 2019)

Tabla 1.

Índice de control nutricional (CONUT)

\begin{tabular}{lllll}
\hline Parámetro & Normal & Leve & Moderado & Severo \\
\hline Albúmina (g/dl) & $3.5-4.5$ & $3.0-3.49$ & $2.5-2.9$ & $<2.5$ \\
$\begin{array}{l}\text { Puntaje } \\
\text { Linfocitos }\end{array}$ & 0 & 2 & 4 & 6 \\
to- & $>1600$ & $1200-15999$ & $800-1199$ & $<800$ \\
$\begin{array}{llll}\text { Puntaje } \\
\text { Colesterol }\end{array}$ & 0 & & & \\
dl) & $>180$ & 1 & 2 & 3 \\
Puntaje & 0 & $140-180$ & $100-139$ & $<100$ \\
Puntaje total & 0 & 1 & & \\
\hline
\end{tabular}

Riesgo de malnutrición: sin riesgo 0-1, leve 2-4, moderado 5-8, Grave $>8$ 
La escala GNRI (Bouillanne O. et al., 2005) ajustada al paciente anciano se calcula con la fórmula (1.489 X albúmina $(\mathrm{g} / \mathrm{L}))+(41.7 \mathrm{X}(\mathrm{Peso} / \mathrm{WLo}))$ donde WLo (Peso ideal calculado con la ecuación de Lorentz): Hombre: $\mathrm{T}-100-((\mathrm{T}-150) / 4)$ y Mujer: $\mathrm{T}-100-((\mathrm{T}-150) / 2.5)$, donde $\mathrm{T}$ : talla en cm, se clasificaron 4 grados [Tabla 2].

Tabla 2.

Clasificación de la Escala Riesgo Nutricional Geriátrico

\begin{tabular}{ll}
\hline Riesgo mayor & GNRI $<82$ \\
Riesgo moderado & GNRI $82-<92$ \\
Bajo riesgo & GNRI 92- $<98$ \\
Sin riesgo & GNRI $>98$ \\
\hline
\end{tabular}

Se formaron dos grupos iniciales para el análisis comparativo: el Grupo A con menos de 7 días de ventilación mecánica y el Grupo B con más de 7 días de ventilación.

Las variables categóricas se analizaron con $\chi^{2}$ y la prueba exacta de Fisher; las variables continuas con prueba de t de Student. Se usó U de Mann-Whitney para variables de distribución anormal, se consideró la significancia estadística con un valor de $\mathrm{p}<0.05$.

Consideraciones éticas: este estudio fue aprobado por el Comité de Investigación y el Comité de Ética de la Investigación del Centro Médico Dalinde, Se autorizó la revisión de expedientes, los datos se hicieron anónimos, codificados según un código alfanumérico que los hizo no identificables para su posterior análisis estadístico, se realizó de acuerdo con los estándares éticos mostrados en la declaración de Helsinki de 2013.

\section{Resultados}

Se incluyeron 68 sujetos, de los cuales el 51.5\% (35) fueron del sexo masculino y 48.5\% (33) del género femenino, con una mediana de edad de 81 años RIQ [72-86], la mediana del IMC fue de 25.9 RIQ [22.0-28.8], con una mayor proporción de sujetos en sobrepeso y obesidad (36.8\% y 20.6\%). Más de la mitad de los sujetos procedieron del servicio de urgencias (58.5\%), seguido de hospitalización (22\%), las comorbilidades más frecuentes fueron hipertensión arterial sistémica (79.4\%), seguida de diabetes mellitus tipo 2 (41.2\%) Uno de cada 3 sujetos tuvo como diagnóstico de ingreso sepsis (32.35\%), uno de cada cuatro ingresos a UCI fue un estado posquirúrgico (20.58\%). [Tabla 3 ] 
Martínez Palma, L.A.;Enríquez Santos, D.; Alberti Minutti, P.

Tabla 3.

Características de los sujetos de estudio

\begin{tabular}{|c|c|c|}
\hline Variable & $\mathbf{N}$ & $\%$ \\
\hline Masculino & 35 & 51.5 \\
\hline Femenino & 33 & 48.5 \\
\hline \multicolumn{3}{|l|}{ Servicio de ingreso } \\
\hline Urgencias & 40 & 58.5 \\
\hline Hospitalización & 15 & 22 \\
\hline Quirófano & 13 & 19.5 \\
\hline \multicolumn{3}{|l|}{ Diagnóstico de ingreso } \\
\hline Sepsis & 22 & 32.35 \\
\hline Estado postoperatorio & 14 & 20.58 \\
\hline Falla Ventilatoria & 11 & 16.17 \\
\hline Patología Gastrointestinal & 8 & 11.76 \\
\hline EVC & 8 & 11.76 \\
\hline Politraumatismo & 4 & 5.88 \\
\hline TCE severo & 1 & 1.47 \\
\hline \multicolumn{3}{|l|}{ Índice de masa corporal } \\
\hline Peso bajo & 8 & 11.8 \\
\hline Peso normal & 21 & 30.9 \\
\hline Sobrepeso & 25 & 36.8 \\
\hline Obesidad I & 7 & 10.3 \\
\hline Obesidad II & 2 & 2.9 \\
\hline Obesidad III & 5 & 7.3 \\
\hline \multicolumn{3}{|l|}{ Comorbilidades Asociadas } \\
\hline $\begin{array}{l}\text { Hipertensión arterial sisté- } \\
\text { mica }\end{array}$ & 54 & 79.4 \\
\hline Diabetes Mellitus 2 & 28 & 41.2 \\
\hline $\begin{array}{l}\text { Insuficiencia Cardiaca Cró- } \\
\text { nica }\end{array}$ & 18 & 26.5 \\
\hline Enfermedad Renal Crónica & 14 & 20.6 \\
\hline Cáncer & 14 & 20.6 \\
\hline Enfermedad cognitiva & 12 & 17.6 \\
\hline Enfermedad tiroidea & 11 & 16.2 \\
\hline
\end{tabular}

$\mathrm{N}$ : 68. \%: porcentaje calculado con base al total de la población. TCE: traumatisno craneoencefálico. EVC: enfermedad vacular cerebral 
Respecto al estado nutricional la albúmina tuvo una mediana de $2.54 \mathrm{~g} / \mathrm{dL}$, las escalas de GNRI y CONUT tuvieron medianas de puntuación de 87 [RIQ 75-96] y 8 [RIQ 6-10] respectivamente. La mediana de días de ventilación mecánica fue de 5 [RIQ 3-8], con una estancia promedio en la UCI de 10. [RIQ 7-16]. (Tabla 4).

Tabla 4.

Variables Cuantitativas

\begin{tabular}{lc}
\hline \multicolumn{1}{c}{ Variable } & Md RIQ \\
\hline Días de VM & $5[3-8]$ \\
Días de estancia & $10[7-16]$ \\
Edad (años) & $81[72-86]$ \\
IMC & $25.5[22.0-28.8]$ \\
Albúmina * & $2.5[2.1-3.0]$ \\
Linfocitos & $915[476-1492]$ \\
Colesterol* & $115[88-145]$ \\
Pre albúmina & $10[8-17]$ \\
Transferrina & $134[97-157]$ \\
Lorentz & $57[52-65]$ \\
APACHE II & $22[14-26]$ \\
SOFA & $8[5-9]$ \\
GNRI & $87[75-96]$ \\
CONUT & $8[6-10]$
\end{tabular}

Md: Mediana. RIQ: Rango inter-cuartílico. VM: ventilación mecánica. IMC: Índice de masa corporal. APACHE II: Acute Physiology and Chronic Health disease Classification System II. SOFA: Sequential Organ Failure Assessment Score. GNRI: Geriatric Nutritional Risk Index. CONUT: Controlling Nutritional Status.

*Variable con distribución normal, representada con Media \pm Desviación Estándar

Durante el análisis bivariado se observó en el grupo A una mediana de edad de 81 años [RIQ 74-86] y mediana de 83 años para el grupo B. La mediana de días de estancia en la Unidad de Cuidados Intensivos en el grupo A fue de 8 días y 20 días para el grupo de más de 7 días de ventilación mecánica, los días a los que estuvieron sometidos a ventilación mecánica en el grupo A fue de 4 días. [RIQ 2-5]. (Tabla 5). 
Martínez Palma, L.A.;Enríquez Santos, D.; Alberti Minutti, P.

Tabla 5.

Análisis bivariado entre los grupos $A$ y $B$

\begin{tabular}{llll}
\hline Variable n (\%) & GRUPO A & GRUPO B & \multicolumn{1}{c}{$\boldsymbol{P}$} \\
\hline Femenino & $24(49)$ & $9(47.4)$ & $0.905^{\mathrm{a}}$ \\
Masculino & $25(51)$ & $10(52.2)$ & \\
Peso Bajo & $6(12.2)$ & $2(10.5)$ & $0.151^{\mathrm{a}}$ \\
Peso Normal & $18(36.7)$ & $3(15.8)$ & \\
Sobrepeso & $16(32.7)$ & $9(47.4)$ & \\
Obesidad I & $5(10.2)$ & $2(10.5)$ & \\
Obesidad II & $4(8.2)$ & $2(10.5)$ & \\
Obesidad III & $0(0)$ & $1(5.3)$ & \\
HAS & $38(77.6)$ & $16(84.2)$ & $0.403^{\mathrm{b}}$ \\
DM2 & $21(42.9)$ & $7(36.8)$ & $0.651^{\mathrm{a}}$ \\
ERC & $11(22.4)$ & $3(15.8)$ & $0.403^{\mathrm{b}}$ \\
ICC & $16(32.7)$ & $2(10.5)$ & $0.074^{\mathrm{b}}$ \\
Cáncer & $11(22.4)$ & $3(15.8)$ & $0.724^{\mathrm{b}}$ \\
Cirugía Reciente & $10(20.4)$ & $4(21.1)$ & $0.953^{\mathrm{a}}$ \\
Enfermedad tiroidea & $4(21.1)$ & $0.497^{\mathrm{a}}$ \\
$7(14.3)$ & & & \\
Enfermedad & $8(16.3)$ & $4(21.1)$ & $0.646^{\mathrm{a}}$ \\
Cognitiva & & & \\
\hline
\end{tabular}

${ }^{\mathrm{a}}=$ Chi Cuadrada de Pearson; ${ }^{\mathrm{b}}=$ Test exacto de Fisher. N: $68 . \%$ : porcentaje calculado con base al total de la población. HAS: hipertensión arterial sistémica. DM2: diabetes mellitus tipo 2. ERC: enfermedad renal crónica. ICC: insuficiencia cardiaca crónica

Los resultados de los parámetros nutricionales al ingreso revelaron albúmina de $2.6 \mathrm{~g} / \mathrm{dL} \pm 0.7$ en el grupo A, $2.4 \mathrm{~g} / \mathrm{dL} \pm 0.5$ para el grupo $\mathrm{B}$, los predictores de mortalidad al ingreso de SOFA fue de 8 [5-9] en el grupo A, para el grupo B 8 [5-11], APACHE II para el grupo de menos de 7 días de ventilación mecánica 22 [13-26] en contraste el conjunto B 22 [17-25], no tuvieron diferencia estadísticamente significativa. El grupo A presentó un puntaje de GNRI 90 [74-97], mientras que el grupo B una mediana de 84 puntos de GNRI [75-93] p 0.432, en contraste la escala de CONUT para el grupo A 8 [6-9] y el grupo B 9 [7-11] p 0.135. (Tabla 6) 
Tabla 6.

Análisis bivariado entre los grupos $A$ y $B$

\begin{tabular}{lccc}
\hline \multicolumn{1}{c}{$\begin{array}{c}\text { Variable Md } \\
\text { [RIQ] }\end{array}$} & Grupo A & Grupo B & $\boldsymbol{P}$ \\
\hline Días de VM & $4[2-5]$ & $12[8-14]$ & $<0.001^{\mathrm{a}}$ \\
Días de estancia & $8[6-12]$ & $20[14-27]$ & $<0.001^{\mathrm{a}}$ \\
Edad (años) & $81[74-86]$ & $83[66-86]$ & $0.989^{\mathrm{a}}$ \\
IMC & $25.2[21.6-28.0]$ & $25.5[23.8-30.1]$ & $0.283^{\mathrm{a}}$ \\
Albúmina* & $2.6[2.1-3.0]$ & $2.4[2.0-2.6]$ & $0.121^{\mathrm{a}}$ \\
Linfocitos & $990[457-1571]$ & $886[538-1378]$ & $0.748^{\mathrm{a}}$ \\
Colesterol* & $112[83-144]$ & $133[91-153]$ & $0.311^{\mathrm{a}}$ \\
Pre-Albúmina & $10[8-16]$ & $10[8-18]$ & $0.847^{\mathrm{a}}$ \\
Transferrina* & $131[92-153]$ & $147[112-172]$ & $0.153^{\mathrm{a}}$ \\
Lorentz & $56[52-63]$ & $60[50-67]$ & $0.446^{\mathrm{a}}$ \\
APACHE II & $22[13-26]$ & $22[17-25]$ & $0.800^{\mathrm{a}}$ \\
SOFA & $8[5-9]$ & $8[5-11]$ & $0.470^{\mathrm{a}}$ \\
GNRI & $90[74-97]$ & $84[75-93]$ & $0.432^{\mathrm{a}}$ \\
CONUT & $8[6-9]$ & $9[7-11]$ & $0.135^{\mathrm{a}}$ \\
\hline
\end{tabular}

a = U de Mann-Whitney. VM: ventilación mecánica. IMC: índice de masa corporal. APACHE II: Acute Physiology and Chronic Health disease Classification System II. SOFA: Sequential Organ Failure Assessment Score. GNRI: Geriatric Nutritional Risk Index. CONUT: Controlling Nutritional Status

\section{Discusión}

Los adultos mayores son portadores de una alta prevalencia de enfermedades crónicas y deterioro funcional lo que aumenta el riesgo de presentar enfermedades críticas, el envejecimiento poblacional pronostica un aumento en la demanda de este grupo poblacional para su ingreso a la UCI, (Yanping Ye. et al., 2017) la ventilación mecánica es uno de los pilares de tratamiento en las Unidades de Cuidados Intensivos, cuando se combina un proceso de enfermedad aguda, los pacientes de edad avanzada en la UCI se encuentran en condiciones excepcionales para presentar un alto riesgo de deterioro nutricional. (Boumendil A. et al., 2007)

Nuestro estudio reportó a la sepsis, falla ventilatoria y estado postoperatorio como los principales motivos de ingreso, lo que correlaciona con los datos de Chen y su grupo de investigación, (Chin-Ming Chen. et al., 2014) existen datos de investigaciones nacionales que nos permiten apuntar que la mayoría de los adultos mayores ingresan a la UCI con una o más enfermedades crónico degenerativas, demostrado en el estudio de González Escudero y su grupo de colaboradores (Eduardo Alberto González Escudero. et al., 2020) donde la hipertensión arterial, Diabetes y enfermedad renal crónica poseen mayor prevalencia.

Además de las múltiples comorbilidades, los pacientes son portadores de síndromes geriátricos como 
la desnutrición y sarcopenia, la información obtenida en nuestra investigación concuerdan con los datos internacionales que sugieren una prevalencia de desnutrición de hasta el $71 \%$ en los adultos mayores ingresados a la UCI, (de Luis D. et al., 2006) aunque estos datos varían dependiendo de la herramienta para su cribado, en el estudio EuroOOPS (Sorensen J. et al., 2008) estos porcentajes se incrementaron hasta $87-100 \%$ de los pacientes admitidos a la UCI.

La valoración nutricional se recomienda que sea realizada de manera rutinaria a su ingreso o al menos durante su estancia en la UCI con el fin de detectar desnutrición e iniciar de manera temprana una terapia dirigida a satisfacer sus necesidades nutricionales.

Los pacientes geriátricos ingresados a la UCI son particularmente difíciles de estadificar porque la mayoría de los criterios diagnósticos no son aplicables al paciente crítico, por lo que es suficiente el cuestionario de tamizaje y la sospecha clínica para detectar e iniciar alguna acción terapéutica; ya que la sarcopenia (Cerri A. P. et al., 2015) se asoció con aumento de la mortalidad a tres meses del egreso hospitalario. $(\mathrm{p}<0.001)$

Nuestro estudio sugiere que los pacientes que requirieron ventilación prolongada al momento de su ingreso; obtuvieron puntajes catalogados como desnutridos severos en la escala de CONUT y GNRI 84 puntos, clasificado como riesgo moderado $(\mathrm{p}=0.432)$, en contraste el grupo con menos de 7 días de ventilación mecánica obtuvieron una escala de GNRI 90 [74-97]; clasificado como un riesgo nutricional moderado o leve, estadificando con la escala de CONUT se obtuvo en promedio 8 puntos definidos como un riesgo nutricional moderado $(p=0.135)$ lo que denota que a mayor riesgo nutricional a menudo se requieren más días de ventilación mecánica.

Sin embargo, estos datos deben ser interpretados con cautela, existen estudios realizados por diferentes autores (Cereda E. et al., 2008; Bouillanne O. et al., 2005) en donde se describe que patologías como infecciones severas, cáncer o enfermedad renal crónica deben de excluirse al utilizar GNRI ya que los niveles de albúmina sérica están alterados sin tener relación con el estado nutricional, patologías que tienen una prevalencia no despreciable en la población de adultos mayores.

Otro factor que debe tomarse en cuenta según nuestra experiencia es el cálculo del peso del paciente al ingreso, ya que puede ser inexacto en el entorno de la UCI debido a la administración de líquidos durante la reanimación inicial.

Hay que resaltar que uno de los principales diagnósticos de ingreso a nuestra UCI es pacientes posquirúrgicos los cuales probablemente sean menos complejos para retiro de ventilación mecánica.

El uso prolongado de ventilación mecánica en adultos mayores confiere un mayor deterioro funcional, así como mayor institucionalización (Chelluri L. et al., 2004), lo que repercute negativamente en la calidad de vida del paciente.

\section{Conclusiones}

La asistencia del adulto mayor en la Unidad de Cuidados Intensivos es una realidad, resulta compleja la evaluación del estado nutricional durante la enfermedad crítica, el estado nutricional valorado con GNRI y CONUT podría demostrar una relación con la duración de la ventilación mecánica. 
El paciente geriátrico debido a los cambios fisiológicos de la edad y sus múltiples comorbilidades se presenta con algún grado de desnutrición. Se demuestra en este estudio que la mayoría de los adultos mayores valorados con GNRI y CONUT presentan un riesgo moderado a severo de desnutrición.

La relación entre el estado nutricional y los días de ventilación mecánica está modulada además por otras variables como la gravedad de la enfermedad de manera que el uso del estado nutricional por sí solo no es suficiente para predecir si el adulto mayor se someterá a ventilación mecánica prolongada.

Limitaciones del estudio: el tipo de estudio retrospectivo y el número de muestra limitan la interpretación de los resultados. Además, la población fue de un centro hospitalario. Por lo tanto, la escalas GNRI y CONUT debe ser validada en un estudio prospectivo y multicéntrico para establecer su mejor papel en la práctica clínica.

\section{Declaratoria de disponibilidad de datos}

Los autores declaran que los datos utilizados en la investigación están disponibles a demanda, existe restricción de acceso ya que la información proviene de expedientes clínicos tanto electrónicos como físicos.

\section{Referencias}

A. J. Bowyer, C. F. Royse (2015) Postoperative recovery and outcomes - what are we measuring and for whom?, Anaesthesia. http://doi.org/10.1111/anae.13312

Abbott, A. L., Silvestrini, M., Topakian, R., Golledge, J., Brunser, A. M., de Borst, G. J., Harbaugh, R. E., Doubal, F. N., Rundek, T., Thapar, A., Davies, A. H., Kam, A., \& Wardlaw, J. M. (2017). Optimizing the Definitions of Stroke, Transient Ischemic Attack, and Infarction for Research and Application in Clinical Practice. Frontiers in neurology, 8, 537. https://doi.org/10.3389/fneur.2017.00537

Alfonso López-Soto, Emilio Sacanella, Juan Manuel Pérez Castejón, José M. Nicolás (2009) El anciano en la unidad de cuidados intensivos, Revista Española de Geriatría y Gerontología. http://doi. org/10.1016/j.regg.2009.03.013

American Diabetes Association (2019). Standards of Medical Care in Diabetes-2019 Abridged for Primary Care Providers. Clinical diabetes: a publication of the American Diabetes Association, 37(1), 11-34. https://doi.org/10.2337/cd18-0105

Angus D. C. (2017). Admitting Elderly Patients to the Intensive Care Unit-Is it the Right Decision? JAMA, 318(15), 1443-1444. https://doi.org/10.1001/jama.2017.14535

B. Polla, G. d'Antona, R. Bottinelli, C. Reggiani. Respiratory muscle fibres: Specialisation and plasticity. (2004). Thorax, 59, pp. 808-817.21. Jaber, S., Petrof, B. J.,

Béduneau, G., Pham, T., Schortgen, F., Piquilloud, L., Zogheib, E., Jonas, M., Grelon, F., Runge, I., Nicolas Terzi, Grangé, S., Barberet, G., Guitard, P. G., Frat, J. P., Constan, A., Chretien, J. M., 
Mancebo, J., Mercat, A., Richard, J. M., Brochard, L., \& WIND (Weaning according to a New Definition) Study Group and the REVA (Réseau Européen de Recherche en Ventilation Artificielle) Network $\$$ (2017). Epidemiology of Weaning Outcome according to a New Definition. The WIND Study. American journal of respiratory and critical care medicine, 195(6), 772-783. https://doi. org/10.1164/rccm.201602-03200C

Bouillanne, O., Morineau, G., Dupont, C., Coulombel, I., Vincent, J. P., Nicolis, I., Benazeth, S., Cynober, L., \& Aussel, C. (2005). Geriatric Nutritional Risk Index: a new index for evaluating at-risk elderly medical patients. The American journal of clinical nutrition, 82(4), 777-783. https://doi. org/10.1093/ajen/82.4.777

Boumendil, A., Somme, D., Garrouste-Orgeas, M., \& Guidet, B. (2007). Should elderly patients be admitted to the intensive care unit?. Intensive care medicine, 33(7), 1252. https://doi.org/10.1007/s00134-007-0621-3

Carrillo-Esper R, De la Torre-León T. (2019) El paciente adulto mayor en la Unidad de Terapia Intensiva. ¿Estamos preparados?, Med Crit.; 33(4):199-203.

Cereda, E., Pusani, C., Limonta, D., \& Vanotti, A. (2008). The association of Geriatric Nutritional Risk Index and total lymphocyte count with short-term nutrition-related complications in institutionalised elderly. Journal of the American College of Nutrition, 27(3), 406-413. https://doi.org/10.1080/07315724.2008 .10719718

Cerri, A. P., Bellelli, G., Mazzone, A., Pittella, F., Landi, F., Zambon, A., \& Annoni, G. (2015). Sarcopenia and malnutrition in acutely ill hospitalized elderly: Prevalence and outcomes. Clinical nutrition (Edinburgh, Scotland), 34(4), 745-751. https://doi.org/10.1016/j.clnu.2014.08.015

Chaker, L., Bianco, A. C., Jonklaas, J., \& Peeters, R. P. (2017). Hypothyroidism. Lancet (London, England), 390(10101), 1550-1562. https://doi.org/10.1016/S0140-6736(17)30703-1

Chakrabarti, B., \& Calverley, P. M. (2016). Management of acute ventilatory failure. Postgraduate medical journal, 82(969), 438-445. https://doi.org/10.1136/pgmj.2005.043208

Chelkeba, L., Mojtahedzadeh, M., \& Mekonnen, Z. (2017). Effect of Calories Delivered on Clinical Outcomes in Critically Ill Patients: Systemic Review and Meta-analysis. Indian journal of critical care medicine: peer-reviewed, official publication of Indian Society of Critical Care Medicine, 21(6), 376-390. https://doi.org/10.4103/ijccm.IJCCM_453_16

Chelluri, L., Im, K. A., Belle, S. H., Schulz, R., Rotondi, A. J., Donahoe, M. P., Sirio, C. A., Mendelsohn, A. B., \& Pinsky, M. R. (2004). Long-term mortality and quality of life after prolonged mechanical ventilation. Critical care medicine, 32(1), 61-69. https://doi.org/10.1097/01.CCM.0000098029.65347. $\underline{\text { F9 }}$

Chin-Ming Chen, Kuo-Chen Cheng, Khee-Siang Chan, Wen-Liang Yu (2014) Age May Not Influence the Outcome of Patients with Severe Sepsis in Intensive Care Units. International Journal of Gerontology. http://doi.org/10.1016/j.ijge.2013.08.004

Cunningham, E. L., McGuinness, B., Herron, B., \& Passmore, A. P. (2015). Dementia. The Ulster medical journal, 84(2), 79-87. 
Eduardo Alberto González Escudero, Jesús Salvador Sánchez Díaz, Armando Solórzano Guerra, Karla Gabriela Peniche Moguel, Josué Eli Villegas Domínguez, María Verónica Calyeca Sánchez (2020) Factores asociados con la mortalidad en el adulto mayor con choque séptico Medicina Crítica. http://doi.org/10.35366/93965

Funk, G. C., Anders, S., Breyer, M. K., Burghuber, O. C., Edelmann, G., Heindl, W., Hinterholzer, G., Kohansal, R., Schuster, R., Schwarzmaier-D’Assie, A., Valentin, A., \& Hartl, S. (2010). Incidence and outcome of weaning from mechanical ventilation according to new categories. The European respiratory journal, 35(1), 88-94. https://doi.org/10.1183/09031936.00056909

Grippa, R. B., Silva, P. S., Barbosa, E., Bresolin, N. L., Mehta, N. M., \& Moreno, Y. M. (2017). Nutritional status as a predictor of duration of mechanical ventilation in critically ill children. Nutrition (Burbank, Los Angeles County, Calif.), 33, 91-95. https://doi.org/10.1016/j.nut.2016.05.002.

Grosu, H. B., Lee, Y. I., Lee, J., Eden, E., Eikermann, M., \& Rose, K. M. (2012). Diaphragm muscle thinning in patients who are mechanically ventilated. Chest, 142(6), 1455-1460. https://doi. org/10.1378/chest.11-1638

Hermans, G., Agten, A., Testelmans, D., Decramer, M., \& Gayan-Ramirez, G. (2010). Increased duration of mechanical ventilation is associated with decreased diaphragmatic force: a prospective observational study. Critical care (London, England), 14(4), R127. https://doi.org/10.1186/cc9094

Ibrahim, E. H., Mehringer, L., Prentice, D., Sherman, G., Schaiff, R., Fraser, V., \& Kollef, M. H. (2002). Early versus late enteral feeding of mechanically ventilated patients: results of a clinical trial. JPEN. Journal of parenteral and enteral nutrition, 26(3), 174-181. https://doi. org/10.1177/0148607102026003174

Ignacio de Ulíbarri, J., González-Madroño, A., Villar, NG de, González, P., González, B., Mancha, A., Rodríguez, F., \& Fernández, G. (2005). CONUT: A tool for Controlling Nutritional Status. First validation in a hospital population. Nutrición Hospitalaria, 20(1), 38-45.

Instituto Nacional de las Mujeres. (2015). Boletín estadístico. Situación de las personas adultas mayores en México. INMUJERES, 1, 1-39. http://cedoc.inmujeres.gob.mx/target= blank

J.L. de-León-Rendón, R.Y. López-Pérez, N.I. Gracida-Mancilla, B. Jiménez-Bobadilla, L. Alarcón-Bernés, S. Mendoza-Ramírez, J.A. Villanueva-Herrero. (2020) La escala control nutricional (CONUT): una herramienta prometedora para el cribado nutricional y como predictor de severidad en la colitis ulcerosa crónica idiopática, Revista de Gastroenterología de México. http://doi.org/10.1016/j. rgmx.2020.05.012

Jung, B., Chanques, G., Berthet, J. P., Rabuel, C., Bouyabrine, H., Courouble, P. Koechlin-Ramonatxo, C., Sebbane, M., Similowski, T., Scheuermann, V., Mebazaa, A., Capdevila, X., Mornet, D., Mercier, J., Lacampagne, A., Philips, A., \& Matecki, S. (2011). Rapidly progressive diaphragmatic weakness and injury during mechanical ventilation in humans. American journal of respiratory and critical care medicine, 183(3), 364-371. https://doi.org/10.1164/rccm.201004-06700C

López Espuela, F., Roncero-Martín, R., Zamorano, J., Rey-Sanchez, P., Aliaga-Vera, I., Portilla Cuenca, 
J. C., Naranjo, I. C., Morán-García, J. M., \& Lavado-García, J. M. (2019). Controlling Nutritional Status (CONUT) Score as a Predictor of All-Cause Mortality at 3 Months in Stroke Patients. Biological research for nursing, 21(5), 564-570. https://doi.org/10.1177/1099800419860253

de Luis, D., Lopez Guzman, A., \& Nutrition Group of Society of Cstilla-Leon (Endocrinology, Diabetes and Nutrition) (2006). Nutritional status of adult patients admitted to internal medicine departments in public hospitals in Castilla y Leon, Spain - A multi-center study. European journal of internal medicine, 17(8), 556-560. https://doi.org/10.1016/j.ejim.2006.02.030

Magda C. Díaz, Gustavo A. Ospina-Tascón, Blanca C. Salazar C (2014) Disfunción muscular respiratoria: una entidad multicausal en el paciente críticamente enfermo sometido a ventilación mecánica Archivos de Bronconeumología. http://doi.org/10.1016/j.arbres.2013.03.005

Le Maguet, P., Roquilly, A., Lasocki, S., Asehnoune, K., Carise, E., Saint Martin, M., Mimoz, O., Le Gac, G., Somme, D., Cattenoz, C., Feuillet, F., Malledant, Y., \& Seguin, P. (2014). Prevalence and impact of frailty on mortality in elderly ICU patients: a prospective, multicenter, observational study. Intensive care medicine, 40(5), 674-682. Marik PE. Should age limit admission to the intensive care unit? The American Journal of Hospice \& Palliative Care. 2007 Feb-Mar; 24(1):63-66. Doi: https://doi.org/10.1007/s00134-014-3253-49.

Mervyn Singer, Clifford S. Deutschman, Christopher Warren Seymour, Manu Shankar-Hari, Djillali Annane, Michael Bauer, Rinaldo Bellomo, Gordon R. Bernard, Jean-Daniel Chiche, Craig M. Coopersmith, Richard S. Hotchkiss, Mitchell M. Levy, John C. Marshall, Greg S. Martin, Steven M. Opal, Gordon D. Rubenfeld, Tom van der Poll, Jean-Louis Vincent, Derek C. Angus (2016) The Third International Consensus Definitions for Sepsis and Septic Shock (Sepsis-3). http://doi. org/10.1001/jama.2016.0287

Nancy Carney, Annette M. Totten, Cindy O’Reilly, Jamie S. Ullman, Gregory W.J. Hawryluk, Michael J. Bell, Susan L. Bratton, Randall Chesnut, Odette A. Harris, Niranjan Kissoon, Andres M. Rubiano, Lori Shutter, Robert C. Tasker, Monica S. Vavilala, Jack Wilberger, David W. Wright, Jamshid Ghajar (2016) Guidelines for the Management of Severe Traumatic Brain Injury, Fourth Edition, Neurosurgery. http://doi.org/10.1227/neu.0000000000001432

Norma Oficial Mexicana NOM-167-SSA1-1997.

Nuttall F. Q. (2015). Body Mass Index: Obesity, BMI, and Health: A Critical Review. Nutrition today, 50(3), 117-128. https://doi.org/10.1097/NT.0000000000000092.

Rau, C. S., Wu, S. C., Kuo, P. J., Chen, Y. C., Chien, P. C., Hsieh, H. Y., \& Hsieh, C. H. (2017). Polytrauma Defined by the New Berlin Definition: A Validation Test Based on Propensity-Score Matching Approach. International journal of environmental research and public health, 14(9), 1045. https:// doi.org/10.3390/ijerph14091045.

Reyes, J. C., Alonso, J. V., Fonseca, J., Santos, M. L., Jiménez, M., \& Braniff, J. (2016). Characteristics and mortality of elderly patients admitted to the Intensive Care Unit of a district hospital. Indian journal of critical care medicine: peer-reviewed, official publication of Indian Society of Critical Care Medicine, 20(7), 391-397. https://doi.org/10.4103/0972-5229.186219 
Rosas-Peralta, M., Borrayo-Sánchez, G., Madrid-Miller, A., Ramírez-Arias, E., \& Pérez-Rodríguez, G. (2016). Hipertensión arterial sistémica en el adulto mayor. Recomendaciones para la práctica clínica [Systemic arterial hypertension in the elderly. Recommendations for clinical practice]. Revista médica del Instituto Mexicano del Seguro Social, 54 Suppl 1, s75-s77.

Sánchez Zúñiga, M. J., \& Carrillo Esper, R. (2018). PROPUESTA PARA LA IMPLEMENTACION DE UN COMITÉ RELACIONADO A LOS CUIDADOS INTENSIVOS EN EL ADULTO MAYOR. El adulto mayor en la unidad de cuidados intensivos, Colegio Mexicano de Medicina Crítica. https://commec.org/wp-content/uploads/2019/08/Terapia-intensiva.pdf

Sorensen, J., Kondrup, J., Prokopowicz, J., Schiesser, M., Krähenbühl, L., Meier, R., Liberda, M., \& EuroOOPS study group (2008). EuroOOPS: an international, multicentre study to implement nutritional risk screening and evaluate clinical outcome. Clinical nutrition (Edinburgh, Scotland), 27(3), 340-349. https://doi.org/10.1016/j.clnu.2008.03.012

Suárez Núñez, L., Rodríguez Felipe, B., Gutiérrez Gutiérrez, L., \& Brito Cruz, A. (2018). Sobre el comportamiento del Índice CONUT de Control Nutricional en el paciente atendido en un hospital general provincial debido a complicaciones quirúrgicas. Revista Cubana De Alimentación Y Nutrición, 28(2), 13.

Suzuki, S., Kanaji, S., Yamamoto, M., Oshikiri, T., Nakamura, T., \& Kakeji, Y. (2019). Controlling Nutritional Status (CONUT) Score Predicts Outcomes of Curative Resection for Gastric Cancer in the Elderly. World journal of surgery, 43(4), 1076-1084. https://doi.org/10.1007/s00268-018-04889-6

Thomas, R., Kanso, A., \& Sedor, J. R. (2008). Chronic kidney disease and its complications. Primary care, 35(2), 329-vii. https://doi.org/10.1016/j.pop.2008.01.008

Vassilakopoulos, T., \& Petrof, B. J. (2004). Ventilator-induced diaphragmatic dysfunction. American journal of respiratory and critical care medicine, 169(3), 336-341. https://doi.org/10.1164/rccm.200304-489CP.

Villagordoa, M. J. (2007). Definición de envejecimiento y síndrome de fragilidad, características epidemiológicas del envejecimiento en México. Revista de Endocrinología y Nutrición, 15(1), 27-31. https://www.medigraphic.com/cgi-bin/new/resumen.cgi?IDARTICULO=12044

Wallace, J. I., Schwartz, R. S., LaCroix, A. Z., Uhlmann, R. F., \& Pearlman, R. A. (1995). Involuntary weight loss in older outpatients: incidence and clinical significance. Journal of the American Geriatrics Society, 43(4), 329-337. https://doi.org/10.1111/j.1532-5415.1995.tb05803.x

Yanping Ye, Bo Zhu, Li Jiang, Qi Jiang, Meiping Wang, Lin Hua, Xiuming Xi (2017) A Contemporary Assessment of Acute Mechanical Ventilation in Beijing Critical Care Medicine. http://doi. org/10.1097/ccm.0000000000002360 